\title{
Fast Non-rigid Multimodal Image Registration Using Local Frequency Maps
}

\author{
B.C. Vemuri and J. Liu \\ Department of Computer and Information Science and Engineering \\ University of Florida \\ vemuri|jliu@cise.ufl.edu
}

\begin{abstract}
In this paper, we present a novel and computationally efficient multimodal image registration scheme that is capable of handling non-rigidly misaligned multi-modal data sets. The algorithm is based on a level-set based image registration algorithm applied to local frequency representations of the input images. The algorithm has been tested on several data sets and we present one of these examples.
\end{abstract}

\section{Introduction}

In this paper, we present a novel method for non-rigidly registering multi-modal images. Our method involves first deriving a brightness "invariant" representation of the image and then using the level-set curve evolution technique for image registration introduced in Vemuri et.al., [1] on these "invariant" representations of the input images.

\section{Local Frequency Representation \& Matching}

The Gabor Filter is a well-known quadrature filter. It achieves the theoretical lower bound of the uncertainty principle. Local frequency computation can be achieved by Gabor filtering the input data and then computing the gradient of local phase followed by summing the squared magnitude of local frequency maps over a discrete set of orientations for a fixed frequency 2. We present and example local frequency representation in figure 2 which depicts a pair of T1 \& T2 MR slices and their corresponding local frequency maps.

Given two local frequency maps $F_{1}(X)$ and $F_{2}(X)$, we want to find the nonrigid transformation between them. This can be achieved by decomposing the non-rigid deformation into incremental rigid transformation and using a moving grid implementation of the following evolution equation:

$$
\boldsymbol{V}_{\boldsymbol{t}}=\left[F_{2}(X)-F_{1}(\boldsymbol{V}(X)] \frac{\nabla F_{1}(\boldsymbol{V}(X))}{\left\|\nabla F_{1}(\boldsymbol{V}(X))\right\|} \text { with } \quad \boldsymbol{V}(X, 0)=\mathbf{0}\right.
$$

where $\boldsymbol{V}=(u, v, w)^{T}$ is the displacement vector at $X$ and the operation $\boldsymbol{V}(X)=$ $(x-u, y-v, z-w)$. For an efficient implementation of this equation, see [1]. 


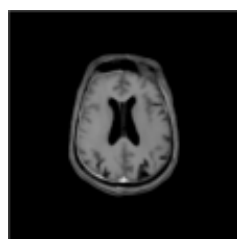

(a)

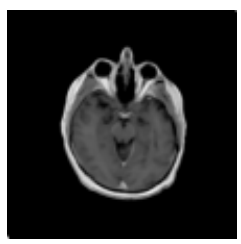

(b)

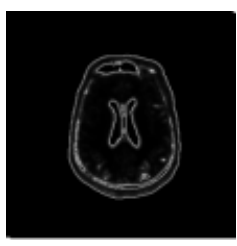

(c)

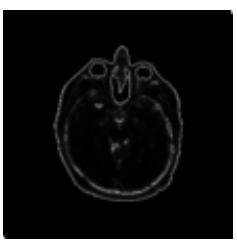

(d)

Fig. 1. (a) T1-weighted MR, (b) T2-weighted MR slices, (c) and (d), corresponding slices of local frequency maps.

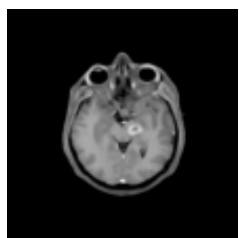

(a)

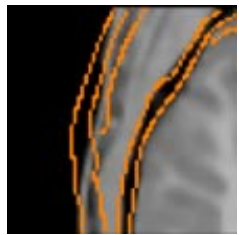

(e)

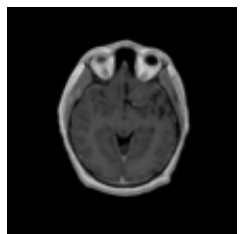

(b)

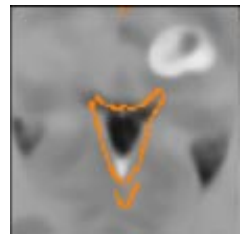

(f)

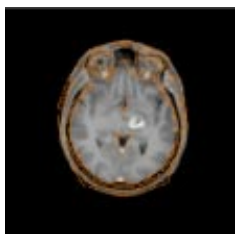

(c)

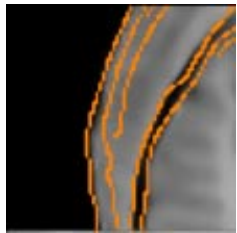

(g)

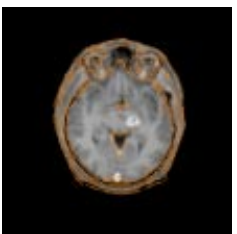

(d)

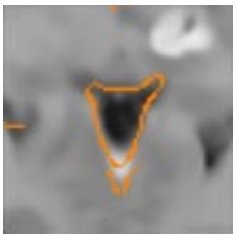

(h)

Fig. 2. Non-Rigid Registration Example: (a) source image, (b) target image, (c) globally transformed source image with superimposed target edge map, (d) non-rigidly transformed source with superimposed target edge map. The second row is the zoom-in version of (c) and (d): (e) left upper and (f) central parts of (c); (g) left upper (h) central parts of $(\mathrm{d})$.

\section{Implementation Results}

We report a registration experiment with a pair of MR images obtained using a T1 and T2 weighted imaging protocols respectively. Each image was obtained from different subjects making it apt for the application of a non-rigid deformation. Figure 2 shows the registration results for the T1 and T2 weighted MR brain scans being registered. Fig. 2(a) and (b) depict the source and target images to be registered. Prior to application of the non-rigid registration, we rigidly align the data sets. Figure 2(c) depicts the globally transformed source and the locally transformed source image is shown in fig. 2(d). 2(c) and 2(d) have been superimposed with the edge map of target image to visually demonstrate the accuracy of the registration. Figures 2(e) and 2(f) depict the residual mis-alignment after the application of the global rigid transform to the source 
using a close up view at two different locations in the source. Figures $2(\mathrm{~g})$ and $2(\mathrm{~h})$ depict the close up view of the alignment achieved after the application of a non-rigid deformation to the globally transformed source image at the same locations as in 2(e) and 2(f). As is evident, the mis-alignment has been dramatically reduced after the application of the non-rigid deformation. The average CPU time taken for computing the local frequency map from the input image is 366 sec. and that for estimating the global rigid motion is $37 \mathrm{sec}$. on an R10000 single CPU of an SGI Onyx. The time taken to compute non-rigid deformation is $357 \mathrm{sec}$, which is much faster than most of the reported non-rigid registration schemes in literature.

\section{Summary}

In this paper, we presented a novel and efficient non-rigid multimodal image registration scheme. The algorithm involved constructing local frequency maps of the input data followed by an application of the level-set based non-rigid registration scheme [1]. The algorithm was tested on several data and illustrated via one example.

\section{Acknowledgments}

We thank Drs. Bova \& Bouchet and Mr. Moore for providing the image data. This research was partially supported by the grants NSF IIS9811042 and NIH RO1-RR13197.

\section{References}

1.B. C. Vemuri et.al., [2000], "A Level-set based approach to image registration," IEEE Workshop on MMBIA, June 10-12, Hilton Head, SC, pp. 86-93.

2.B. C. Vemuri, J. Liu, J. L. Marroquin, [2001], "Robust Multimodal Image Registration using Local Frequency Representation," IPMI'01, June 18-22, Davis, CA, pp. 176-182. 